\title{
ON RINGS ALL OF WHOSE FACTOR RINGS ARE INTEGRAL DOMAINS
}

\author{
YASUYUKI HIRANO
}

(Received 7 January 1991)

Communicated by P. Schultz

\begin{abstract}
A ring $R$ is called a (proper) quotient no-zero-divisor ring if every (proper) nonzero factor ring of $R$ has no zero-divisors. A characterization of a quotient no-zero-divisor ring is given. Using it, the additive groups of quotient no-zero-divisor rings are determined. In addition, for an arbitrary positive integer $n$, a quotient no-zero-divisor ring with exactly $n$ proper ideals is constructed. Finally, proper quotient no-zero-divisor rings and their additive groups are classified.

1991 Mathematics subject classification (Amer. Math. Soc.): primary 16 A 45; secondary 16 A 02,16 A 48 .
\end{abstract}

\section{Introduction}

As in Feigelstock [1], a ring $R$ is called a quotient no-zero-divisor ring if every homomorphic image of $R$ has no zero-divisors. Similarly a ring $R$ is called a proper quotient no-zero-divisor ring if every proper homomorphic image of $R$ has no zero-divisors. Feigelstock [1, Question 4.1.13] asked: What can be said about the quotient no-zero-divisor rings and the proper quotient no-zero-divisor rings? In this paper, we consider this question. We first give a characterization of a quotient no-zero-divisor ring. As a corollary, we obtain that the additive group of a quotient no-zero-divisor ring is either a direct sum of copies of the additive group of the field of rational numbers or a direct sum of cyclic groups of order $p$ for some fixed prime $p$. In addition, we show that a quotient no-

(C) 1993 Australian Mathematical Society 0263-6115/93 \$A2.00+0.00 
zero-divisor ring satisfying a polynomial identity is a division ring. We next construct a quotient no-zero-divisor ring with exactly $n$ proper ideals for an arbitrary positive integer $n$. We do not know whether there exists a quotient no-zero-divisor ring with infinitely many ideals or not. However we give an example of a Hausdorff topological ring $R$ such that $R$ has infinitely many closed proper ideals and all of them are completely prime. In the second half of this paper, we study the proper quotient no-zero-divisor rings. We classify the proper quotient no-zero-divisor rings into four types and determine their structures. As a result, we describe the structure of the additive groups of proper quotient no-zero-divisor rings.

\section{Quotient no-zero-divisor rings}

A proper ideal $P$ of a ring $R$ is said to be completely prime if $R / P$ has no zerodivisors. Thus $R$ is a quotient no-zero-divisor ring if and only if every proper ideal of $R$ is completely prime. For each element $a$ of a ring $R,(a)$ denotes the principal ideal of $R$ generated by $a$, that is, $(a)=R a+a R+R a R+\mathbb{Z} a$. A ring $R$ is called a chain ring if the lattice of ideals of $R$ is totally ordered. We shall now give a characterization of a quotient no-zero-divisor ring.

THEOREM 1. The following statements are equivalent:

(1) $R$ is a quotient no-zero-divisor ring;

(2) $R$ is a chain ring satisfying $(a)=\left(a^{2}\right)$ for all elements $a$ in $R$.

PROOF. (1) $\Rightarrow(2)$. Let $a$ be an element of $R$. If $\left(a^{2}\right)=R$, then clearly we have $(a)=\left(a^{2}\right)$. So suppose that $\left(a^{2}\right) \neq R$. Then $\left(a^{2}\right)$ is completely prime by hypothesis, and so we get $a \in\left(a^{2}\right)$. Therefore we have $(a)=\left(a^{2}\right)$. To prove that $R$ is a chain ring, let $I, J$ be two ideals of $R$. Assume, to the contrary, that $I \not \subset J$ and $J \not \subset I$ and take $a \in I \backslash J$ and $b \in J \backslash I$. Then we see that $a b \in I \cap J$, and so $I \cap J$ is not completely prime. This is contrary to our hypothesis. Thus $R$ is a chain ring.

(2) $\Rightarrow(1)$. Let $P$ be a proper ideal of $R$. To prove that $P$ is completely prime, let $a, b$ be two elements of $R$ such that $a b \in P$. Then, for any $x \in R \cup \mathbb{Z}$, we have $b x a \in(b x a)=\left((b x a)^{2}\right) \subset(a b) \subset P$. Hence $(b)(a) \subset P$. Since $R$ is a chain ring, without loss of generality, we may assume that $(b) \subset(a)$. Then (b) $=\left(b^{2}\right) \subset(b)(b) \subset(b)(a) \subset P$, that is, $b \in P$. This proves that $P$ is a completely prime ideal. 
Let $\mathbb{Q}^{+}$denote the additive group of the field of rational numbers, and for any positive integer $n$, let $\mathbb{Z}(n)$ denote the cyclic group of order $n$. We shall determine the additive groups of quotient no-zero-divisor rings.

COROLLARY 1. Let $G$ be an abelian group. Then the following statements are equivalent:

(1) $G$ is the additive group of a quotient no-zero-divisor ring;

(2) $G$ is isomorphic to either $\bigoplus_{\alpha} \mathbb{Q}^{+}$or $\bigoplus_{\alpha} \mathbb{Z}(p)$ for some prime $p$, where $\alpha$ is an arbitrary cardinal.

PROOF. (1) $\Rightarrow(2)$. Suppose that $G$ is the additive group of a quotient nozero-divisor ring $R$. Since $R$ is an integral domain, the characteristic of $R$ is either 0 or a prime $p$. In the latter case, $R$ is a vector space over a field of order $p$, and hence $G \cong \bigoplus_{\alpha} \mathbb{Z}(p)$ for some cardinal $\alpha$. Suppose now that $R$ is of characteristic 0 . Take an element $a \in R$ and a positive integer $n$. Then, by Theorem 1, $n a \in\left((n a)^{2}\right)=n^{2}\left(a^{2}\right)$. Since $R$ is of characteristic 0 , we conclude that $a \in n R$. This implies that the additive group of $R$ is a torsion-free divisible group. By Fuchs [2, Theorem 23.1], $G \cong \bigoplus_{\alpha} \mathbb{Q}^{+}$for some cardinal $\alpha$.

(2) $\Rightarrow(1)$. Needless to say, all fields are quotient no-zero-divisor rings. If $G \cong \bigoplus_{\alpha} \mathbb{Q}^{+}$, then $G$ is the additive group of a field extension of degree $\alpha$ of the field of rational numbers. On the other hand, if $G \cong \bigoplus_{\alpha} \mathbb{Z}(p)$, then $G$ is the additive group of a field extension of degree $\alpha$ of a field of order $p$. This completes the proof.

The first Weyl algebra $A_{1}(\mathbb{Q})$ over the field $\mathbb{Q}$ of rational numbers is the algebra on $x, y$ over $\mathbb{Q}$ with the defining relation $x y-y x=1$. It is well known that $A_{1}(\mathbb{Q})$ is a simple domain with unity 1 . It is easy to check that $R=A_{1}(\mathbb{Q}) x$ is a simple domain without unity. Hence $R$ is an example of a quotient no-zero-divisor ring without unity. As another corollary of Theorem 1 , we have the following.

COROLLARY 2. Let $R$ be a quotient no-zero-divisor ring and let $C$ denote the center of $R$. Then $R$ has a unity if and only if $C \neq 0$.

PROOF. Suppose that $C \neq 0$, and take a nonzero element $a$ of $C$. By Theorem 1, we have $(a)=\left(a^{2}\right)$. Then we can write $a=a e$ for some $e \in(a)$. Then, for any $x \in R$, we have $a(x-x e)=x a-x a e=0$. Similarly, we have $a(x-e x)=0$. Since $R$ has no zero-divisors, these imply that $x=x e=e x$ for all $x \in R$. Hence $e$ is a unity of $R$. 
It is easy to see that a commutative quotient no-zero-divisor ring is a field. More generally, we have the following.

PROPOSITION 1. Let $R$ be a quotient no-zero-divisor ring. If $R$ satisfies a polynomial identity, then $R$ is a division ring.

Proof. Let $C$ denote the center of $R$. Since $R$ is prime, $C \neq 0$ by Herstein [3, Theorem 1.4.2]. Hence, by Corollary $1, R$ has a unity 1. Let $a$ be a nonzero element of $C$. By Theorem $1,(a)=\left(a^{2}\right)$. Hence there exists $b \in R$ such that $a=a^{2} b$. Since $R$ is an integral domain, $b$ is the inverse of $a$. Clearly $b$ is in $C$. Hence $C$ is a field. By Rowen [6, Corollary 1.6.28], $R$ is a simple Artinian ring. Since $R$ has no zero-divisors, $R$ must be a division ring.

Let $R$ be a chain ring. If the number of proper ideals of $R$ is $n$, then $R$ is called an $n$-chain ring. We shall show that there exist $n$-chain quotient no-zero-divisor rings for any positive integer $n$. Note that a 1-chain quotient no-zero-divisor ring is nothing but a simple domain. Let $K$ be a field and let $A$ be an algebra over $K$. Then, for each positive integer $m$, we let $T_{K}^{m}(A)=A \otimes_{K} \cdots \otimes_{K} A$ (tensor product taken $m$ times). To construct $n$-chain quotient no-zero-divisor rings, we use a simple domain $D$ with center $K$ such that $T_{K}^{m}(D)$ is an integral domain for any positive integer $m$. We shall now give some examples of such simple domains.

EXAMPLE 1. (1) Let $K$ be a field of characteristic 0 . Then, for each positive integer $n$, the $n$-th Weyl algebra $A_{n}(K)$ is a simple domain with 1 which is not a division ring. The center of $A_{n}(K)$ is $K$, and $T_{k}^{m}\left(A_{n}(k)\right) \cong A_{m n}(K)$. Hence $T_{K}^{m}\left(A_{n}(K)\right)$ is an integral domain for any positive integer $m$.

(2) Let $F$ be a field (of any characteristic) and let $F(\lambda)$ be the field of rational functions in $\lambda$ over $F$. Let $B=B(\lambda, F(\lambda))$ be a ring defined as the $F(\lambda)$-algebra generated by $x, x^{-1}, y$ and $y^{-1}$ subject to the relation $x y=\lambda y x$. By Jategaonkar [4, Theorem 2.1], $B$ is a simple domain with 1 which is not a division ring. Clearly the center of $B$ is $F(\lambda)$. Since $T_{F(\lambda)}^{m}(B)=B\left(\lambda, T_{F(\lambda)}^{m-1}(B)\right)$, using induction on $m$ and Jategaonkar [4, Proposition 1.1(a)], we can show that $T_{F(\lambda)}^{m}(B)$ is an integral domain for any positive integer $m$.

THEOREM 2. Let $D$ be a simple domain with 1 which is not a division ring, and let $K$ denote the center of $D$. Suppose that $T_{K}^{m}(D)$ is an integral domain for any positive integer $m$. Take a non-unit $0 \neq x \in D$ and let $R_{1}=K+x D$. 
Define a subalgebra $R_{n}$ of $T_{K}^{n}(D)$ inductively: $R_{n}=K+$ ax $D \otimes_{K} R_{n-1}$. Then $R_{n}$ is an $(n+1)$-chain quotient no-zero-divisor ring.

PROOF. Let $I$ be a nonzero proper ideal of $R_{1}$. Since $D$ is a simple domain, we see that $I \supset x D I x D=x D$. Since $R_{1} / x D$ is isomorphic to the field $K$, $x D$ is a maximal ideal of $R_{1}$, and hence we conclude that $I=x D$. Thus 0 , $x D$ and $R_{1}$ are all the ideals of $R_{1}$. Obviously, 0 and $x D$ are completely prime. Therefore $R_{1}$ is a 2-chain quotient no-zero-divisor ring. By induction on $n$, we shall prove that the proper ideals of $R_{n}$ are $0, T_{K}^{n}(x D), T_{K}^{n-1}(x D) \otimes_{K} R_{1}$, $T_{K}^{n-2}(x D) \otimes_{K} R_{2}, \ldots, x D \otimes_{K} R_{n-1}$ and these are completely prime ideals. Let us set $R_{0}=K$. Assume now that $n>1$ and that the proper ideals of $R_{n-1}$ are $I_{0}=$ $0, I_{1}=T_{K}^{n-1}(x D), \ldots, I_{n-1}=x D \otimes_{K} R_{n-2}$ and these are completely prime. Since $D$ is a simple ring with center $K$, by Renault [5, Theorem 5.1.3] the proper ideals of $D \otimes_{K} R_{n-1}$ are $D \otimes_{K} I_{0}=0, D \otimes_{K} I_{1}, \ldots, D \otimes_{K} I_{n-1}$. Let $I$ be a proper ideal of $R_{n}$. Then $\left(D \otimes-K R_{n-1}\right) I\left(x D \otimes_{K} R_{n-1}\right)=D \otimes_{K} I_{m}$ for some $m<n$. Then we obtain that $(x \otimes 1) I(x \otimes 1) \subset x D \otimes_{K} I_{m}$. Since $R_{n} /\left(x D \otimes_{K} I_{m}\right) \cong$ $K+x D \otimes_{K}\left(R_{n-1} / I_{m}\right) \cong K+x D \otimes_{K} R_{n-m-1}=R_{n-m}, x D \otimes_{K} I_{m}$ is completely prime by the induction hypothesis. Since $(x \otimes 1) I(x \otimes 1) \subset x D \otimes_{K} I_{m} \subset I$ and $x \otimes 1 \notin x D \otimes_{K} I_{m}$, we conclude that $I=x D \otimes_{K} I_{m}$, and hence $I$ is a completely prime ideal of $R_{n}$. If $m \neq 0$, then $I=x D \otimes_{K} T_{K}^{n-m}(x D) \otimes_{K} R_{m-1}=$ $T_{K}^{n-m+1}(x D) \otimes_{K} R_{m-1}$, and if $m=0$, then $I=0$. This completes the induction.

We do not know whether there exists a quotient no-zero-divisor ring with infinitely many ideals or not. However we shall construct a Hausdorff topological ring $R$ such that $R$ has infinitely many closed proper ideals and all of them are completely prime.

Let $D$ be a simple domain with center $K$ satisfying the hypothesis of Theorem $2, R_{n}(n \geq 1)$ be the rings defined in Theorem 2 using $D$ and let $R_{0}=K$. Let $n$ be a positive integer, and $I_{n}$ the unique minimal ideal of $R_{n}$. As shown in the proof of Theorem 2, we have a canonical isomorphism $R_{n} / I \cong R_{n-1}$. Let $f_{n}: R_{n} \rightarrow R_{n-1}$ be the epimorphism induced by this isomorphism. Then $\left\{R_{n}, f_{n}\right\}$ is an inverse system of rings.

THEOREM 3. The inverse limit $S=\lim _{\leftarrow} R_{n}$ becomes a Hausdorff topological ring all of whose closed proper ideals are completely prime.

PROOF. Let $p_{n}: S \rightarrow R_{n}$ denote the canonical projection, $M_{-1}=S$, and $M_{n}=\operatorname{Ker} p_{n}$ for each $n \geq 0$. By taking $\left\{M_{n}\right\}$ to be a base of neighborhoods of $0, S$ becomes a topological ring. Since $\bigcap_{n \geq-1} M_{n}=0, S$ is Hausdorff. Since 
$M_{n}=S-\bigcup_{a \in S \backslash M_{n}}\left(a+M_{n}\right), M_{n}$ is open and closed. Since $S / M_{n} \cong R_{n}$ and each $R_{n}$ is an integral domain, $M_{n}$ is a completely prime ideal for any $n \geq 0$. Now let $M$ be a nonzero closed ideal of $S$. We shall prove that $M=M_{m}$ for some $m \geq-1$. Since $\bigcap_{n \geq-1} M_{n}=0$ and $M_{-1}=S \supset M_{0} \supset M_{1} \supset \ldots$, there exists an integer $m \geq-1$ such that $M_{m} \supset M$ and $M_{m+1} \not \supset M$. Since $S / M_{k} \cong R_{k}$ for each $k$, by taking the structure of the lattice of ideals of $R_{k}$ into consideration, we conclude that $M_{k}+M=M_{m}$ for all $k \geq m$. Therefore $M_{m} \subset M+M_{k}$ for all $k \geq 0$. To prove that $M_{m} \subset M$, let $a \in M_{m}$. Then $a \in M+M_{k}$ for all $k \geq 0$. Hence $\left(a+M_{k}\right) \cap M \neq 0$ for all $k \geq 0$. Since $M$ is closed and $\left\{a+M_{k} \mid k \geq 0\right\}$ is a base of neighborhoods of $a$, we conclude that $a \in M$. This proves that $M=M_{m}$.

\section{Proper quotient no-zero-divisor rings}

We shall classify the proper quotient no-zero-divisor rings.

THEOREM 4. Let $R$ be a ring. Then $R$ is a proper quotient no-zero-divisor ring if and only if one of the following holds:

(a) $R$ is a quotient no-zero-divisor ring;

(b) $R$ is a simple ring with zero-divisors;

(c) $R$ is not an integral domain and $R$ has a unique minimal ideal $P$ such that $R / P$ is a quotient no-zero-divisor ring;

(d) $R$ is the direct sum of two simple domains;

(e) There exist two quotient no-zero-divisor rings $A$ and $B$ with unique proper minimal ideals $P$ and $Q$ respectively, such that there is an isomorphism

$$
\sigma: A / P \rightarrow B / Q \text {, and } R=\{(a, b) \in A \times B \mid \sigma(a+P)=b+Q\} .
$$

PROOF. Let $R$ be a proper quotient no-zero-divisor ring such that 0 is not a completely prime ideal. Suppose first that $R$ is a chain ring. If $R$ has no nonzero proper ideals, then $R$ satisfies (b). So we may assume that $R$ has a nonzero proper ideal. Let $P$ denote the intersection of all nonzero proper ideals of $R$. Since $R$ is a chain ring and since every nonzero proper ideal is completely prime, $P$ is completely prime. Hence $P$ is nonzero by hypothesis. In this case, $R$ satisfies (c). Suppose next that $R$ has two ideals $P$ and $Q$ such that $P \not \subset Q$ and $Q \not \subset P$. Then $R / P \cap Q$ is not an integral domain, and so $P \cap Q$ must be zero. Let $I$ be a nonzero ideal of $R$ different from $P$ and $Q$. Since $R / I$ is an 
integral domain, either $P \subset I$ or $Q \subset I$. Suppose that $P \not \subset I$. Then $Q \subset I$ and $I \cap P \subset P$. If $I \cap P \neq 0$, then $Q \subset I \cap P$, because $P \not \subset I \cap P$. Then $Q=(I \cap P) \cap Q \subset P \cap Q=0$, which is a contradiction. Hence $I \cap P=0 \subset Q$, and so $I=Q$. This contradicts the choice of $I$. Therefore $I$ contains $P$. Similarly we can prove that $I$ contains $Q$. Hence $P+Q$ is minimum among the nonzero ideals of $R$ different from $P$ and $Q$. Now if $P+Q=R$, then $R$ is the direct sum of the simple domains $P$ and $Q$, and so $R$ satisfies (d). So assume that $P+Q \neq R$. Let us set $A=R / Q$ and $B=R / P$. By hypothesis $A$ and $B$ are quotient no-zero-divisor rings. Since $P \cap Q=0, P$ and $Q$ can be viewed as ideals of $A$ and $B$, respectively. By the above observation, $P$ and $Q$ are minimal ideals of $A$ and $B$ respectively. Then we see $A / P \cong R /(P+Q) \cong B / Q$. Let $\sigma: A / P \rightarrow B / Q$ denote this isomorphism. Consider the natural embedding $f: R \rightarrow A \times B ; f(a)=(a+Q, a+P)$ for all $a \in R$. Let $S$ denote the set $\{(x, y) \in A \times B \mid \sigma(x+P)=y+Q\}$. We shall show that $\operatorname{Im}(f)=S$. Clearly $\operatorname{Im}(f)$ is contained in $S$. Let $(x, y)=(a+Q, b+P) \in S$, where $a$, $b$ are elements of $R$. Then $a+(P+Q)=b+(P+Q)$ in $R /(P+Q)$, that is, $a-b \in P+Q$. Hence we can write $a-b=p+q$ for some $p \in P$ and $q \in Q$. Now it is easy to see that $f(a-q)=(x, y)$. This proves $\operatorname{Im}(f)=S$, and hence $R$ satisfies (e).

Conversely, if $R$ satisfies one of (a)-(d), then clearly $R$ is a proper quotient no-zero-divisor ring. Assume now that $R$ satisfies the condition (e). Then we can easily see that the proper nonzero ideals of $R$ are $(P, 0)=\{(p, 0) \in A \times B \mid$ $p \in P\},(0, Q)$, and

$$
H(I)=\{(a, b) \in A \times B \mid a \in I, \sigma(a+P)=b+Q\},
$$

where $I$ runs over all proper ideals of $A$ containing $P$. Since $R /(P, 0) \cong B$, $R /(0, Q) \cong A$ and $R / H(I) \cong A / I$, all of these nonzero ideals are completely prime. This completes the proof.

We shall give examples of rings satisfying the conditions (c) or (e) in Theorem 4 .

EXAMPLE 2. (1) Let $n>1$ be an integer and let $R$ be an $n$-chain quotient no-zero-divisor ring with unique minimal ideal $P$. Consider the set

$$
S=\left\{\left(\begin{array}{cc}
a & p \\
0 & a
\end{array}\right) \mid a \in R, p \in P\right\} .
$$


By defining addition and multiplication in $S$ as in ordinary matrices, $S$ becomes a ring. We can easily see that $S$ satisfies the condition (c) in Theorem 4 and $S$ is not a prime ring. On the other hand, let $F$ be a field and let $A$ be the ring of countable matrices over $F$ of the form

$$
\left(\begin{array}{ccccc}
C_{n} & 0 & & & \\
0 & a & & & \\
& & \ddots & & \\
& & & a & \\
& & & & \ddots
\end{array}\right)
$$

where $a \in F$ and $C_{n}$ is an arbitrary $n \times n$ matrix over $F$ and $n$ is allowed to be any integer. Then $A$ is a prime ring satisfying (c) in Theorem 4.

(2) Let $n>1$ be an integer and let $R$ be an $n$-chain quotient no-zero-divisor ring with unique minimal ideal $P$. Then $S=\{(a, b) \in R \times R \mid a-b \in P\}$ is a ring satisfying the condition (e) in Theorem 4.

As a result of Theorem 4, we can describe the structure of the additive groups of proper quotient no-zero-divisor rings.

COROLLARY 3. Let $R$ be a proper quotient no-zero-divisor ring and let $R^{+}$ denote the additive group of $R$. Then one of the following holds:

(1) $R^{+}=\bigoplus_{\alpha} \mathbb{Q}^{+}$where $\alpha$ is a cardinal;

(2) $R^{+}=\bigoplus_{\alpha} \mathbb{Z}(p)$ where $p$ is a prime and $\alpha$ is a cardinal;

(3) $R^{+}=\bigoplus_{\alpha} \mathbb{Q}^{+} \oplus \bigoplus_{\beta} \mathbb{Z}(p)$ is a prime and $\alpha, \beta$ are cardinals;

(4) $R^{+}=\bigoplus_{\alpha} \mathbb{Z}(p) \oplus \bigoplus_{\beta} \mathbb{Z}(q)$ where $p$ and $q$ are distinct primes and $\alpha, \beta$ are cardinals;

(5) $R^{+}=\bigoplus_{\alpha} \mathbb{Z}\left(p^{2}\right)$ where $p$ is a prime and $\alpha$ is a cardinal;

(6) $R^{+}=\bigoplus_{\alpha} \mathbb{Z}(p) \oplus \bigoplus_{\beta} \mathbb{Z}\left(p^{2}\right)$ where $p$ is a prime and $\alpha, \beta$ are cardinals.

PROOF. If $R$ satisfies the condition (a) in Theorem 4, then either (1) or (2) holds by Corollary 1 . Now suppose that $R$ satisfies (b) in Theorem 4 . Then the characteristic of $R$ is either a prime $p$ or 0 . If $\operatorname{char}(R)=p$, then (2) holds. On the other hand, if $\operatorname{char}(R)=0$, then $n R=R$ for any positive integer $n$, because $n R$ is a nonzero ideal of $R$. This implies that $R^{+}$is divisible. Hence (1) holds by Fuchs [2, Theorem 23.1]. Suppose next that $R$ satisfies the condition (c) in Theorem 4. Then $R$ is a chain ring and $P$ is the unique minimal ideal of $R$. If $P^{2} \neq 0$, then $P^{2}=P$. In this case, $R$ is a prime ring and $I^{2}=I$ for any ideals $I$ of $R$, because every ideal of $R / P$ is idempotent by Theorem 1 . If the characteristic of $R$ is a prime $p$, then (2) holds. So 
assume that $\operatorname{char}(R)=0$ and let $n$ be an arbitrary positive integer. Then, since $n R=(n R)^{2}=n^{2} R^{2}=n^{2} R$, we obtain $n R=R$. Then $R^{+}$is a torsion-free divisible group, and hence (1) holds again by Fuchs [2, Theorem 23.1]. In case $P^{2}=0, P$ is a right $R / P$-module. If $\operatorname{char}(R / P)=0$, then $R / P$ is a vector space over $\mathbb{Q}$ by Corollary 1 , and hence the right $R / P$-module $P$ is also a vector space over $\mathbb{Q}$. Then $R^{+}$is isomorphic to $P^{+} \oplus(R / P)^{+}$, and hence (1) holds. On the other hand, if $\operatorname{char}(R / P)=p$, then $\operatorname{char}(R)$ equals either $p$ or $p^{2}$. Then, by Fuchs [2, Theorem 17.2], $R^{+}$satisfies either (2), (5) or (6). If $R$ satisfies (d) in Theorem 4, then $R^{+}$satisfies one of (1)-(4). Finally, suppose that $R$ satisfies the condition (e) in Theorem 4 . Then, by Corollary $1, A$ is a vector space over either $\mathbb{Q}$ or $\mathbb{Z} / p \mathbb{Z}$ for some prime $p$. Similarly $B$ is a vector space over either $\mathbb{Q}$ or $\mathbb{Z} / q \mathbb{Z}$ for some prime $q$. Since $A / P$ is isomorphic to $B / Q$, it holds that $\operatorname{char}(A)=\operatorname{char}(B)$. If $\operatorname{char}(A)=\operatorname{char}(B)=p>0$, then $R^{+}$satisfies (2), because $R$ is a subring of $A \times B$. Suppose now that $A$ and $B$ are vector spaces over $\mathbb{Q}$. Then we can easily see that the isomorphism $\sigma: A / P \rightarrow B / Q$ is a $\mathbb{Q}$-space isomorphism. Therefore $R$ is also a vector space over $\mathbb{Q}$, and hence (1) holds in this case.

REMARK. Let $p$ be a prime. Then $\mathbb{Z} / p^{2} \mathbb{Z}$ is a proper quotient no-zero-divisor ring satisfying (5) of Corollary 3 . However we do not know the existence of a proper quotient no-zero-divisor ring satisfying (6) of Corollary 3.

\section{References}

[1] S. Feigelstock, Additive groups of rings, vol. II, Research Notes in Mathematics 169 (Pitman, Boston, 1988).

[2] L. Fuchs, Infinite abelian groups, vol. I (Academic Press, New York, 1970).

[3] I. N. Herstein, Rings with involution (University of Chicago Press, Chicago, 1976).

[4] V. A. Jategaonkar, 'A multiplicative analog of the Weyl algebra', Comm. Algebra 12 (1984), 1669-1688.

[5] G. Renault, Algèbre non commutative (Gauthier-Villars, Paris, 1975).

[6] L. H. Rowen, Polynomial identities in ring theory (Academic Press, New York, 1980).

Okayama University

Okayama, 700

Japan 